\title{
Responsibility and Blame: A Structural-Model Approach
}

\author{
Hana Chockler \\ College of Computer and Information Science \\ Northeastern University, Boston 02115 MA, USA \\ www.ccs.neu.edu/home/hanac \\ Joseph Y. Halpern \\ Computer Science Department \\ Cornell University, Ithaca, NY 14853, USA \\ www.cs.cornell.edu/home/halpern
}

HANAC@CCS.NEU.EDU

HALPERN@CS.CORNELL.EDU

\begin{abstract}
Causality is typically treated an all-or-nothing concept; either $A$ is a cause of $B$ or it is not. We extend the definition of causality introduced by Halpern and Pearl (2004a) to take into account the degree of responsibility of $A$ for $B$. For example, if someone wins an election 11-0, then each person who votes for him is less responsible for the victory than if he had won 6-5. We then define a notion of degree of blame, which takes into account an agent's epistemic state. Roughly speaking, the degree of blame of $A$ for $B$ is the expected degree of responsibility of $A$ for $B$, taken over the epistemic state of an agent.
\end{abstract}

\section{Introduction}

There have been many attempts to define causality going back to Hume (1739), and continuing to the present (see, for example, (Collins, Hall, \& Paul, 2004; Pearl, 2000) for some recent work). While many definitions of causality have been proposed, all of them treat causality as an all-or-nothing concept. That is, $A$ is either a cause of $B$ or it is not. As a consequence, thinking only in terms of causality does not at times allow us to make distinctions that we may want to make. For example, suppose that Mr. B wins an election against Mr. G by a vote of 11-0. Each of the people who voted for Mr. B is a cause of him winning. However, it seems that their degree of responsibility should not be as great as in the case when Mr. B wins 6-5.

In this paper, we present a definition of responsibility that takes this distinction into account. The definition is an extension of a definition of causality introduced by Halpern and Pearl (2004a). Like many other definitions of causality going back to Hume (1739), this definition is based on counterfactual dependence. Roughly speaking, $A$ is a cause of $B$ if, had $A$ not happened (this is the counterfactual condition, since $A \operatorname{did}$ in fact happen) then $B$ would not have happened. As is well known, this naive definition does not capture all the subtleties involved with causality. (If it did, there would be far fewer papers in the philosophy literature!) In the case of the $6-5$ vote, it is clear that, according to this definition, each of the voters for Mr. B is a cause of him winning, since if they had voted against Mr. B, he would have lost. On the other hand, in the case of the 11-0 vote, there are no causes according to the naive counterfactual definition. A change of one vote does not make a difference. Indeed, in this case, we do say in natural language that the cause is somewhat "diffuse". 
While in this case the standard counterfactual definition may not seem quite so problematic, the following example (taken from (Hall, 2004)) shows that things can be even more subtle. Suppose that Suzy and Billy both pick up rocks and throw them at a bottle. Suzy's rock gets there first, shattering the bottle. Since both throws are perfectly accurate, Billy's would have shattered the bottle had Suzy not thrown. Thus, according to the naive counterfactual definition, Suzy's throw is not a cause of the bottle shattering. This certainly seems counter to intuition.

Both problems are dealt with the same way by Halpern and Pearl (2004a). Roughly speaking, the idea is that $A$ is a cause of $B$ if $B$ counterfactually depends on $C$ under some contingency. For example, voter 1 is a cause of Mr. B winning even if the vote is 11-0 because, under the contingency that 5 of the other voters had voted for Mr. G instead, voter 1's vote would have become critical; if he had then changed his vote, Mr. B would not have won. Similarly, Suzy's throw is the cause of the bottle shattering because the bottle shattering counterfactually depends on Suzy's throw, under the contingency that Billy doesn't throw. (There are further subtleties in the definition that guarantee that, if things are modeled appropriately, Billy's throw is not a cause. These are discussed in Section 2.)

It is precisely this consideration of contingencies that lets us define degree of responsibility. We take the degree of responsibility of $A$ for $B$ to be $1 /(N+1)$, where $N$ is the minimal number of changes that have to be made to obtain a contingency where $B$ counterfactually depends on $A$. (If $A$ is not a cause of $B$, then the degree of responsibility is 0 .) In particular, this means that in the case of the 11-0 vote, the degree of responsibility of any voter for the victory is $1 / 6$, since 5 changes have to be made before a vote is critical. If the vote were 1001-0, the degree of responsibility of any voter would be $1 / 501$. On the other hand, if the vote is 5-4, then the degree of responsibility of each voter for Mr. B for Mr. B's victory is 1 ; each voter is critical. As we would expect, those voters who voted for Mr. G have degree of responsibility 0 for Mr. B's victory, since they are not causes of the victory. Finally, in the case of Suzy and Billy, even though Suzy is the only cause of the bottle shattering, Suzy's degree of responsibility is $1 / 2$, while Billy's is 0 . Thus, the degree of responsibility measures to some extent whether or not there are other potential causes.

When determining responsibility, it is assumed that everything relevant about the facts of the world and how the world works (which we characterize in terms of what are called structural equations) is known. For example, when saying that voter 1 has degree of responsibility $1 / 6$ for Mr. B's win when the vote is $11-0$, we assume that the vote and the procedure for determining a winner (majority wins) is known. There is no uncertainty about this. Just as with causality, there is no difficulty in talking about the probability that someone has a certain degree of responsibility by putting a probability distribution on the way the world could be and how it works. But this misses out on important component of determining what we call here blame: the epistemic state. Consider a doctor who treats a patient with a particular drug resulting in the patient's death. The doctor's treatment is a cause of the patient's death; indeed, the doctor may well bear degree of responsibility 1 for the death. However, if the doctor had no idea that the treatment had adverse side effects for people with high blood pressure, he should perhaps not be held to blame for the death. Actually, in legal arguments, it may not be so relevant what the doctor actually did or did not know, but what he should have known. Thus, rather than considering the 
doctor's actual epistemic state, it may be more important to consider what his epistemic state should have been. But, in any case, if we are trying to determine whether the doctor is to blame for the patient's death, we must take into account the doctor's epistemic state.

We present a definition of blame that considers whether agent $a$ performing action $b$ is to blame for an outcome $\varphi$. The definition is relative to an epistemic state for $a$, which is taken, roughly speaking, to be a set of situations before action $b$ is performed, together with a probability on them. The degree of blame is then essentially the expected degree of responsibility of action $b$ for $\varphi$ (except that we ignore situations where $\varphi$ was already true or $b$ was already performed). To understand the difference between responsibility and blame, suppose that there is a firing squad consisting of ten excellent marksmen. Only one of them has live bullets in his rifle; the rest have blanks. The marksmen do not know which of them has the live bullets. The marksmen shoot at the prisoner and he dies. The only marksman that is the cause of the prisoner's death is the one with the live bullets. That marksman has degree of responsibility 1 for the death; all the rest have degree of responsibility 0 . However, each of the marksmen has degree of blame $1 / 10 .^{1}$

While we believe that our definitions of responsibility and blame are reasonable, they certainly do not capture all the connotations of these words as used in the literature. In the philosophy literature, papers on responsibility typically are concerned with moral responsibility (see, for example, (Zimmerman, 1988)). Our definitions, by design, do not take into account intentions or possible alternative actions, both of which seem necessary in dealing with moral issues. For example, there is no question that Truman was in part responsible and to blame for the deaths resulting from dropping the atom bombs on Hiroshima and Nagasaki. However, to decide whether this is a morally reprehensible act, it is also necessary to consider the alternative actions he could have performed, and their possible outcomes. While our definitions do not directly address these moral issues, we believe that they may be helpful in elucidating them. Shafer (2001) discusses a notion of responsibility that seems somewhat in the spirit of our notion of blame, especially in that he views responsibility as being based (in part) on causality. However, he does not give a formal definition of responsibility, so it is hard to compare our definitions to his. However, there are some significant technical differences between his notion of causality (discussed in (Shafer, 1996)) and that on which our notions are based. We suspect that any notion of responsibility or blame that he would define would be different from ours. We return to these issues in Section 5 .

The rest of this paper is organized as follows. In Section 2 we review the basic definitions of causal models based on structural equations, which are the basis for our definitions of responsibility and blame. In Section 3, we review the definition of causality from (Halpern \& Pearl, 2004a), and show how it can be modified to give a definition of responsibility. We show that the definition of responsibility gives reasonable answer in a number of cases, and briefly discuss how it can be used in program verification (see (Chockler, Halpern, \& Kupferman, 2003)). In Section 3.3, we give our definition of blame. In Section 4, we discuss the complexity of computing responsibility and blame. We conclude in Section 5 with some discussion of responsibility and blame. Proofs are deferred to the appendix.

1. We thank Tim Williamson for this example. 


\section{Causal Models: A Review}

In this section, we review the details of the definitions of causal models from (Halpern \& Pearl, 2004a). This section is essentially identical to the corresponding section in (Chockler et al., 2003); the material is largely taken from (Halpern \& Pearl, 2004a).

A signature is a tuple $\mathcal{S}=\langle\mathcal{U}, \mathcal{V}, \mathcal{R}\rangle$, where $\mathcal{U}$ is a finite set of exogenous variables, $\mathcal{V}$ is a finite set of endogenous variables, and $\mathcal{R}$ associates with every variable $Y \in \mathcal{U} \cup \mathcal{V}$ a finite nonempty set $\mathcal{R}(Y)$ of possible values for $Y$. Intuitively, the exogenous variables are ones whose values are determined by factors outside the model, while the endogenous variables are ones whose values are ultimately determined by the exogenous variables. A causal model over signature $\mathcal{S}$ is a tuple $M=\langle\mathcal{S}, \mathcal{F}\rangle$, where $\mathcal{F}$ associates with every endogenous variable $X \in \mathcal{V}$ a function $F_{X}$ such that $F_{X}:\left(\times_{U \in \mathcal{U}} \mathcal{R}(U) \times\left(\times_{Y \in \mathcal{V} \backslash\{X\}} \mathcal{R}(Y)\right)\right) \rightarrow \mathcal{R}(X)$. That is, $F_{X}$ describes how the value of the endogenous variable $X$ is determined by the values of all other variables in $\mathcal{U} \cup \mathcal{V}$. If $\mathcal{R}(Y)$ contains only two values for each $Y \in \mathcal{U} \cup \mathcal{V}$, then we say that $M$ is a binary causal model.

We can describe (some salient features of) a causal model $M$ using a causal network. This is a graph with nodes corresponding to the random variables in $\mathcal{V}$ and an edge from a node labeled $X$ to one labeled $Y$ if $F_{Y}$ depends on the value of $X$. Intuitively, variables can have a causal effect only on their descendants in the causal network; if $Y$ is not a descendant of $X$, then a change in the value of $X$ has no affect on the value of $Y$. For ease of exposition, we restrict attention to what are called recursive models. These are ones whose associated causal network is a directed acyclic graph (that is, a graph that has no cycle of edges). Actually, it suffices for our purposes that, for each setting $\vec{u}$ for the variables in $\mathcal{U}$, there is no cycle among the edges of the causal network. We call a setting $\vec{u}$ for the variables in $\mathcal{U}$ a context. It should be clear that if $M$ is a recursive causal model, then there is always a unique solution to the equations in $M$, given a context.

The equations determined by $\left\{F_{X}: X \in \mathcal{V}\right\}$ can be thought of as representing processes (or mechanisms) by which values are assigned to variables. For example, if $F_{X}(Y, Z, U)=$ $Y+U$ (which we usually write as $X=Y+U$ ), then if $Y=3$ and $U=2$, then $X=5$, regardless of how $Z$ is set. This equation also gives counterfactual information. It says that, in the context $U=4$, if $Y$ were 4 , then $X$ would be 8 , regardless of what value $X$ and $Z$ actually take in the real world. That is, if $U=4$ and the value of $Y$ were forced to be 4 (regardless of its actual value), then the value of $X$ would be 8 .

While the equations for a given problem are typically obvious, the choice of variables may not be. For example, consider the rock-throwing example from the introduction. In this case, a naive model might have an exogenous variable $U$ that encapsulates whatever background factors cause Suzy and Billy to decide to throw the rock (the details of $U$ do not matter, since we are interested only in the context where $U$ 's value is such that both Suzy and Billy throw), a variable $S T$ for Suzy throws $(S T=1$ if Suzy throws, and $S T=0$ if she doesn't), a variable $B T$ for Billy throws, and a variable $B S$ for bottle shatters. In the naive model, whose graph is given in Figure $1, B S$ is 1 if one of $S T$ and $B T$ is 1 . (Note that the graph omits the exogenous variable $U$, since it plays no role. In the graph, there is an arrow from variable $X$ to variable $Y$ if the value of $Y$ depends on the value of $X$.)

This causal model does not distinguish between Suzy and Billy's rocks hitting the bottle simultaneously and Suzy's rock hitting first. A more sophisticated model might also include 


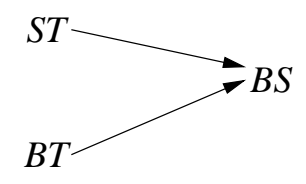

Figure 1: A naive model for the rock-throwing example.

variables $S H$ and $B H$, for Suzy's rock hits the bottle and Billy's rock hits the bottle. Clearly $B S$ is 1 iff one of $S H$ and $B H$ is 1 . However, now, $S H$ is 1 if $S T$ is 1 , and $B H=1$ if $B T=1$ and $S H=0$. Thus, Billy's throw hits if Billy throws and Suzy's rock doesn't hit. This model is described by the following graph, where we implicitly assume a context where Suzy throws first, so there is an edge from $S H$ to $B H$, but not one in the other direction.

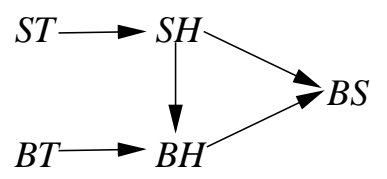

Figure 2: A better model for the rock-throwing example.

Given a causal model $M=(\mathcal{S}, \mathcal{F})$, a (possibly empty) vector $\vec{X}$ of variables in $\mathcal{V}$, and vectors $\vec{x}$ and $\vec{u}$ of values for the variables in $\vec{X}$ and $\mathcal{U}$, respectively, we can define a new causal model denoted $M_{\vec{X} \leftarrow \vec{x}}$ over the signature $\mathcal{S}_{\vec{X}}=\left(\mathcal{U}, \mathcal{V}-\vec{X},\left.\mathcal{R}\right|_{\mathcal{V}-\vec{X}}\right)$. Formally, $M_{\vec{X} \leftarrow \vec{x}}=\left(\mathcal{S}_{\vec{X}}, \mathcal{F} \vec{X} \leftarrow \vec{x}\right)$, where $F_{Y}^{\vec{X} \leftarrow \vec{x}}$ is obtained from $F_{Y}$ by setting the values of the variables in $\vec{X}$ to $\vec{x}$. Intuitively, this is the causal model that results when the variables in $\vec{X}$ are set to $\vec{x}$ by some external action that affects only the variables in $\vec{X}$; we do not model the action or its causes explicitly. For example, if $M$ is the more sophisticated model for the rock-throwing example, then $M_{S T \leftarrow 0}$ is the model where Suzy doesn't throw.

Given a signature $\mathcal{S}=(\mathcal{U}, \mathcal{V}, \mathcal{R})$, a formula of the form $X=x$, for $X \in \mathcal{V}$ and $x \in \mathcal{R}(X)$, is called a primitive event. A basic causal formula has the form $\left[Y_{1} \leftarrow y_{1}, \ldots, Y_{k} \leftarrow y_{k}\right] \varphi$, where

- $\varphi$ is a Boolean combination of primitive events;

- $Y_{1}, \ldots, Y_{k}$ are distinct variables in $\mathcal{V}$; and

- $y_{i} \in \mathcal{R}\left(Y_{i}\right)$.

Such a formula is abbreviated as $[\vec{Y} \leftarrow \vec{y}] \varphi$. The special case where $k=0$ is abbreviated as $\varphi$. Intuitively, $\left[Y_{1} \leftarrow y_{1}, \ldots, Y_{k} \leftarrow y_{k}\right] \varphi$ says that $\varphi$ holds in the counterfactual world that would arise if $Y_{i}$ is set to $y_{i}, i=1, \ldots, k$. A causal formula is a Boolean combination of basic causal formulas.

A causal formula $\varphi$ is true or false in a causal model, given a context. We write $(M, \vec{u}) \models$ $\varphi$ if $\varphi$ is true in causal model $M$ given context $\vec{u} .(M, \vec{u}) \models[\vec{Y} \leftarrow \vec{y}](X=x)$ if the variable $X$ has value $x$ in the unique (since we are dealing with recursive models) solution to the equations in $M_{\vec{Y} \leftarrow \vec{y}}$ in context $\vec{u}$ (that is, the unique vector of values for the exogenous variables that simultaneously satisfies all equations $F_{Z}^{\vec{Y} \leftarrow \vec{y}}, Z \in \mathcal{V}-\vec{Y}$, with the variables in $\mathcal{U}$ set to $\vec{u}$ ). We extend the definition to arbitrary causal formulas in the obvious way. 


\section{Causality and Responsibility}

\subsection{Causality}

We start with the definition of cause from (Halpern \& Pearl, 2004a).

Definition 3.1 (Cause) We say that $\vec{X}=\vec{x}$ is a cause of $\varphi$ in $(M, \vec{u})$ if the following three conditions hold:

AC1. $(M, \vec{u}) \models(\vec{X}=\vec{x}) \wedge \varphi$.

AC2. There exist a partition $(\vec{Z}, \vec{W})$ of $\mathcal{V}$ with $\vec{X} \subseteq \vec{Z}$ and some setting $\left(\vec{x}^{\prime}, \vec{w}^{\prime}\right)$ of the variables in $(\vec{X}, \vec{W})$ such that if $(M, \vec{u}) \models Z=z^{*}$ for $Z \in \vec{Z}$, then

(a) $(M, \vec{u}) \models\left[\vec{X} \leftarrow \vec{x}^{\prime}, \vec{W} \leftarrow \vec{w}^{\prime}\right] \neg \varphi$. That is, changing $(\vec{X}, \vec{W})$ from $(\vec{x}, \vec{w})$ to $\left(\vec{x}^{\prime}, \vec{w}^{\prime}\right)$ changes $\varphi$ from true to false.

(b) $(M, \vec{u}) \models\left[\vec{X} \leftarrow \vec{x}, \vec{W} \leftarrow \vec{w}^{\prime}, \vec{Z}^{\prime} \leftarrow \vec{z}^{*}\right] \varphi$ for all subsets $\vec{Z}^{\prime}$ of $\vec{Z}-\vec{X}$. That is, setting $\vec{W}$ to $\vec{w}^{\prime}$ should have no effect on $\varphi$ as long as $\vec{X}$ has the value $\vec{x}$, even if all the variables in an arbitrary subset of $\vec{Z}$ are set to their original values in the context $\vec{u}$.

AC3. $(\vec{X}=\vec{x})$ is minimal, that is, no subset of $\vec{X}$ satisfies AC2.

AC1 just says that $A$ cannot be a cause of $B$ unless both $A$ and $B$ are true, while AC3 is a minimality condition to prevent, for example, Suzy throwing the rock and sneezing from being a cause of the bottle shattering. Eiter and Lukasiewicz (2002b) showed that one consequence of AC3 is that causes can always be taken to be single conjuncts. Thus, from here on in, we talk about $X=x$ being the cause of $\varphi$, rather than $\vec{X}=\vec{x}$ being the cause. The core of this definition lies in AC2. Informally, the variables in $\vec{Z}$ should be thought of as describing the "active causal process" from $X$ to $\varphi$. These are the variables that mediate between $X$ and $\varphi$. AC2(a) is reminiscent of the traditional counterfactual criterion, according to which $X=x$ is a cause of $\varphi$ if change the value of $X$ results in $\varphi$ being false. However, $\mathrm{AC} 2(\mathrm{a})$ is more permissive than the traditional criterion; it allows the dependence of $\varphi$ on $X$ to be tested under special structural contingencies, in which the variables $\vec{W}$ are held constant at some setting $\vec{w}^{\prime}$. AC2(b) is an attempt to counteract the "permissiveness" of AC2(a) with regard to structural contingencies. Essentially, it ensures that $X$ alone suffices to bring about the change from $\varphi$ to $\neg \varphi$; setting $\vec{W}$ to $\vec{w}^{\prime}$ merely eliminates spurious side effects that tend to mask the action of $X$.

To understand the role of $\mathrm{AC2}(\mathrm{b})$, consider the rock-throwing example again. In the model in Figure 1, it is easy to see that both Suzy and Billy are causes of the bottle shattering. Taking $\vec{Z}=\{S T, B S\}$, consider the structural contingency where Billy doesn't throw $(B T=0)$. Clearly $[S T \leftarrow 0, B T \leftarrow 0] B S=0$ and $[S T \leftarrow 1, B T \leftarrow 0] B S=1$ both hold, so Suzy is a cause of the bottle shattering. A symmetric argument shows that Billy is also a cause.

But now consider the model described in Figure 2. It is still the case that Suzy is a cause in this model. We can take $\vec{Z}=\{S T, S H, B S\}$ and again consider the contingency where Billy doesn't throw. However, Billy is not a cause of the bottle shattering. For suppose that we now take $\vec{Z}=\{B T, B H, B S\}$ and consider the contingency where Suzy doesn't throw. 
Clearly AC2(a) holds, since if Billy doesn't throw (under this contingency), then the bottle doesn't shatter. However, AC2(b) does not hold. Since $B H \in \vec{Z}$, if we set $B H$ to 0 (it's original value), then $\mathrm{AC} 2(\mathrm{~b})$ requires that $[B T \leftarrow 1, S T \leftarrow 0, B H \leftarrow 0](B S=1)$ hold, but it does not. Similar arguments show that no other choice of $(\vec{Z}, \vec{W})$ makes Billy's throw a cause.

Halpern and Pearl (2004a) also consider a slightly more refined definition of causality, where there is a set of allowable settings for the endogenous settings, and the only contingencies that can be considered in $\mathrm{AC} 2(\mathrm{~b})$ are ones where the settings $\left(\vec{W}=\vec{w}^{\prime}, \vec{X}=\vec{x}^{\prime}\right)$ and $\left(\vec{W}=\vec{w}^{\prime}, \vec{X}=\vec{x}\right)$ are allowable. The intuition here is that we do not want to have causality demonstrated by an "unreasonable" contingency. For example, in the rock throwing example, we may not want to allow a setting where $S T=0, B T=1$, and $B H=0$, since this means that Suzy doesn't throw, Billy does, and yet Billy doesn't hit the bottle; this setting contradicts the assumption that Billy's throw is perfectly accurate. We return to this point later.

\subsection{Responsibility}

The definition of responsibility in causal models extends the definition of causality.

Definition 3.2 (Degree of Responsibility) The degree of responsibility of $X=x$ for $\varphi$ in $(M, \vec{u})$, denoted $\operatorname{dr}((M, \vec{u}),(X=x), \varphi)$, is 0 if $X=x$ is not a cause of $\varphi$ in $(M, \vec{u})$; it is $1 /(k+1)$ if $X=x$ is a cause of $\varphi$ in $(M, \vec{u})$ and there exists a partition $(\vec{Z}, \vec{W})$ and setting $\left(x^{\prime}, \vec{w}^{\prime}\right)$ for which AC2 holds such that (a) $k$ variables in $\vec{W}$ have different values in $\vec{w}^{\prime}$ than they do in the context $\vec{u}$ and (b) there is no partition $\left(\vec{Z}^{\prime}, \vec{W}^{\prime}\right)$ and setting $\left(x^{\prime \prime}, \vec{w}^{\prime \prime}\right)$ satisfying AC2 such that only $k^{\prime}<k$ variables have different values in $\vec{w}^{\prime \prime}$ than they do the context $\vec{u}$.

Intuitively, $d r((M, \vec{u}),(X=x), \varphi)$ measures the minimal number of changes that have to be made in $\vec{u}$ in order to make $\varphi$ counterfactually depend on $X$. If no partition of $\mathcal{V}$ to $(\vec{Z}, \vec{W})$ makes $\varphi$ counterfactually depend on $(X=x)$, then the minimal number of changes in $\vec{u}$ in Definition 3.2 is taken to have cardinality $\infty$, and thus the degree of responsibility of $X=x$ is 0 . If $\varphi$ counterfactually depends on $X=x$, that is, changing the value of $X$ alone falsifies $\varphi$ in $(M, \vec{u})$, then the degree of responsibility of $X=x$ in $\varphi$ is 1 . In other cases the degree of responsibility is strictly between 0 and 1 . Note that $X=x$ is a cause of $\varphi$ iff the degree of responsibility of $X=x$ for $\varphi$ is greater than 0 .

Example 3.3 Consider the voting example from the introduction. Suppose there are 11 voters. Voter $i$ is represented by a variable $X_{i}, i=1, \ldots, 11$; the outcome is represented by the variable $O$, which is 1 if $\mathrm{Mr}$. B wins and 0 if $\mathrm{Mr}$. G wins. In the context where Mr. B wins $11-0$, it is easy to check that each voter is a cause of the victory (that is $X_{i}=1$ is a cause of $O=1$, for $i=1, \ldots, 11)$. However, the degree of responsibility of $X_{i}=1$ for is $O=1$ is just $1 / 6$, since at least five other voters must change their votes before changing $X_{i}$ to 0 results in $O=0$. But now consider the context where Mr. B wins 6-5. Again, each voter who votes for Mr. B is a cause of him winning. However, now each of these voters have degree of responsibility 1 . That is, if $X_{i}=1$, changing $X_{i}$ to 0 is already enough to make $O=0$; no other variables need to change. 
Example 3.4 It is easy to see that Suzy's throw has degree of responsibility $1 / 2$ for the bottle shattering in the naive model described in Figure 1; Suzy's throw becomes critical in the contingency where Billy does not throw. In the more refined model of Figure 2, Suzy's degree of responsibility is 1 . If we take $\vec{W}$ to consist of $\{B T, B H\}$, and keep both variables at their current setting (that is, consider the contingency where $B T=1$ and $B H=0$ ), then Suzy's throw becomes critical; if she throws, the bottle shatters, and if she does not throw, the bottle does not shatter (since $B H=0$ ). As we suggested earlier, the setting $(S T=0, B T=1, B H=0)$ may be a somewhat unreasonable one to consider, since it requires Billy's throw to miss. If the setting $(S T=0, B T=1, B H=0)$ is not allowable, then we cannot consider this contingency. In that case, Suzy's degree of responsibility is again $1 / 2$, since we must consider the contingency where Billy does not throw. Thus, the restriction to allowable settings allows us to capture what seems like a significant intuition here.

As we mentioned in the introduction, in a companion paper (Chockler et al., 2003) we apply our notion of responsibility to program verification. The idea is to determine the degree of responsibility of the setting of each state for the satisfaction of a specification in a given system. For example, given a specification of the form $\diamond p$ (eventually $p$ is true), if $p$ is true in only one state of the verified system, then that state has degree of responsibility 1 for the specification. On the other hand, if $p$ is true in three states, each state only has degree of responsibility $1 / 3$. Experience has shown that if there are many states with low degree of responsibility for a specification, then either the specification is incomplete (perhaps $p$ really did have to happen three times, in which case the specification should have said so), or there is a problem with the system generated by the program, since it has redundant states.

The degree of responsibility can also be used to provide a measure of the degree of fault-tolerance in a system. If a component is critical to an outcome, it will have degree of responsibility 1 . To ensure fault tolerance, we need to make sure that no component has a high degree of responsibility for an outcome. Going back to the example of $\diamond p$, the degree of responsibility of $1 / 3$ for a state means that the system is robust to the simultaneous failures of at most two states.

\subsection{Blame}

The definitions of both causality and responsibility assume that the context and the structural equations are given; there is no uncertainty. We are often interested in assigning a degree of blame to an action. This assignment depends on the epistemic state of the agent before the action was performed. Intuitively, if the agent had no reason to believe, before he performed the action, that his action would result in a particular outcome, then he should not be held to blame for the outcome (even if in fact his action caused the outcome).

There are two significant sources of uncertainty for an agent who is contemplating performing an action:

- what the true situation is (that is, what value various variables have); for example, a doctor may be uncertain about whether a patient has high blood pressure. 
- how the world works; for example, a doctor may be uncertain about the side effects of a given medication;

In our framework, the "true situation" is determined by the context; "how the world works" is determined by the structural equations. We model an agent's uncertainty by a pair $(\mathcal{K}, \operatorname{Pr})$, where $\mathcal{K}$ is a set of pairs of the form $(M, \vec{u})$, where $M$ is a causal model and $\vec{u}$ is a context, and $\operatorname{Pr}$ is a probability distribution over $\mathcal{K}$. Following (Halpern \& Pearl, 2004b), who used such epistemic states in their definition of explanation, we call a pair $(M, \vec{u})$ a situation.

We think of $\mathcal{K}$ as describing the situations that the agent considers possible before $X$ is set to $x$. The degree of blame that setting $X$ to $x$ has for $\varphi$ is then the expected degree of responsibility of $X=x$ for $\varphi$ in $\left(M_{X \leftarrow x}, \vec{u}\right)$, taken over the situations $(M, \vec{u}) \in \mathcal{K}$. Note that the situation $\left(M_{X \leftarrow x}, \vec{u}\right)$ for $(M, \vec{u}) \in \mathcal{K}$ are those that the agent considers possible after $X$ is set to $x$.

Definition 3.5 (Blame) The degree of blame of setting $X$ to $x$ for $\varphi$ relative to epistemic state $(\mathcal{K}, \operatorname{Pr})$, denoted $\operatorname{db}(\mathcal{K}, \operatorname{Pr}, X \leftarrow x, \varphi)$, is

$$
\sum_{(M, \vec{u}) \in \mathcal{K}} \operatorname{dr}\left(\left(M_{X \leftarrow x}, \vec{u}\right), X=x, \varphi\right) \operatorname{Pr}((M, \vec{u})) .
$$

Example 3.6 Suppose that we are trying to compute the degree of blame of Suzy's throwing the rock for the bottle shattering. Suppose that the only causal model that Suzy considers possible is essentially like that of Figure 2, with some minor modifications: $B T$ can now take on three values, say $0,1,2$; as before, if $B T=0$ then Billy doesn't throw, if $B T=1$, then Billy does throw, and if $B T=2$, then Billy throws extra hard. Assume that the causal model is such that if $B T=1$, then Suzy's rock will hit the bottle first, but if $B T=2$, they will hit simultaneously. Thus, $S H=1$ if $S T=1$, and $B H=1$ if $B T=1$ and $S H=0$ or if $B T=2$. Call this structural model $M$.

At time 0 , Suzy considers the following four situations equally likely:

- $\left(M, \vec{u}_{1}\right)$, where $\vec{u}_{1}$ is such that Billy already threw at time 0 (and hence the bottle is shattered);

- $\left(M, \vec{u}_{2}\right)$, where the bottle was whole before Suzy's throw, and Billy throws extra hard, so Billy's throw and Suzy's throw hit the bottle simultaneously (this essentially gives the model in Figure 1);

- $\left(M, \vec{u}_{3}\right)$, where the bottle was whole before Suzy's throw, and Suzy's throw hit before Billy's throw (this essentially gives the model in Figure 2); and

- $\left(M, \vec{u}_{4}\right)$, where the bottle was whole before Suzy's throw, and Billy did not throw.

The bottle is already shattered in $\left(M, \vec{u}_{1}\right)$ before Suzy's action, so Suzy's throw is not a cause of the bottle shattering, and her degree of responsibility for the shattered bottle is 0 . As discussed earlier, the degree of responsibility of Suzy's throw for the bottle shattering is $1 / 2$ in $\left(M, \vec{u}_{2}\right)$ and 1 in both $\left(M, \vec{u}_{3}\right)$ and $\left(M, \vec{u}_{4}\right)$. Thus, the degree of blame is $\frac{1}{4} \cdot \frac{1}{2}+\frac{1}{4} \cdot 1+\frac{1}{4} \cdot 1=$ $\frac{5}{8}$. If we further require that the contingencies in $\mathrm{AC} 2(\mathrm{~b})$ involve only allowable settings, 
and assume that the setting $(S T=0, B T=1, B H=0)$ is not allowable, then the degree of responsibility of Suzy's throw in $\left(M, \vec{u}_{3}\right)$ is $1 / 2$; in this case, the degree of blame is $\frac{1}{4} \cdot \frac{1}{2}+\frac{1}{4} \cdot \frac{1}{2}+\frac{1}{4} \cdot 1=\frac{1}{2}$.

Example 3.7 Consider again the example of the firing squad with ten excellent marksmen. Suppose that marksman 1 knows that exactly one marksman has a live bullet in his rifle, and that all the marksmen will shoot. Thus, he considers 10 situations possible, depending on who has the bullet. Let $p_{i}$ be his prior probability that marksman $i$ has the live bullet. Then the degree of blame (according to marksman 1) of the $i$ th marksman's shot for the death is $p_{i}$. The degree of responsibility is either 1 or 0 , depending on whether or not marksman $i$ actually had the live bullet. Thus, it is possible for the degree of responsibility to be 1 and the degree of blame to be 0 (if marksman 1 mistakenly ascribes probability 0 to his having the live bullet, when in fact he does), and it is possible for the degree of responsibility to be 0 and the degree of blame to be 1 (if he mistakenly ascribes probability 1 to his having the bullet when he in fact does not). The difference between the degree of responsibility and the degree of blame stems from the fact that the degree of responsibility is measured with respect to the true situation, whereas the degree of blame is measured with respect to the agent's epistemic state, in which the true situation may be asigned arbitrary probability.

Example 3.8 The previous example suggests that both degree of blame and degree of responsibility may be relevant in a legal setting. Another issue that is relevant in legal settings is whether to consider actual epistemic state or to consider what the epistemic state should have been. The former is relevant when considering intent. To see the relevance of the latter, consider a patient who dies as a result of being treated by a doctor with a particular drug. Assume that the patient died due to the drug's adverse side effects on people with high blood pressure and, for simplicity, that this was the only cause of death. Suppose that the doctor was not aware of the drug's adverse side effects. (Formally, this means that he does not consider possible a situation with a causal model where taking the drug causes death.) Then, relative to the doctor's actual epistemic state, the doctor's degree of blame will be 0 . However, a lawyer might argue in court that the doctor should have known that treatment had adverse side effects for patients with high blood pressure (because this is well documented in the literature) and thus should have checked the patient's blood pressure. If the doctor had performed this test, he would of course have known that the patient had high blood pressure. With respect to the resulting epistemic state, the doctor's degree of blame for the death is quite high. Of course, the lawyer's job is to convince the court that the latter epistemic state is the appropriate one to consider when assigning degree of blame.

Our definition of blame considers the epistemic state of the agent before the action was performed. It is also of interest to consider the expected degree of responsibility after the action was performed. To understand the differences, again consider the patient who dies as a result of being treated by a doctor with a particular drug. The doctor's epistemic state after the patient's death is likely to be quite different from her epistemic state before the patient's death. She may still consider it possible that the patient died for reasons other than the treatment, but will consider causal structures where the treatment was a cause of 
death more likely. Thus, the doctor will likely have higher degree of blame relative to her epistemic state after the treatment.

Interestingly, all three epistemic states (the epistemic state that an agent actually has before performing an action, the epistemic state that the agent should have had before performing the action, and the epistemic state after performing the action) have been considered relevant to determining responsibility according to different legal theories (Hart \& Honoré, 1985, p. 482).

\section{The Complexity of Computing Responsibility and Blame}

In this section we present complexity results for computing the degree of responsibility and blame for general recursive models.

\subsection{The complexity of computing responsibility}

Complexity results for computing causality were presented by Eiter and Lukasiewicz (2002a, $2002 \mathrm{~b})$. They showed that the problem of detecting whether $X=x$ is an actual cause of $\varphi$ is $\Sigma_{2}^{P}$-complete for general recursive models and NP-complete for binary models (Eiter \& Lukasiewicz, 2002b). (Recall that $\Sigma_{2}^{P}$ is the second level of the polynomial hierarchy and that binary models are ones where all random variables can take on exactly two values.) There is a similar gap between the complexity of computing the degree of responsibility and blame in general models and in binary models.

For a complexity class $A, \mathrm{FP}^{\mathrm{A}[\log n]}$ consists of all functions that can be computed by a polynomial-time Turing machine with an oracle for a problem in $A$, which on input $x$ asks a total of $O(\log |x|)$ queries (cf. (Papadimitriou, 1984)). A function $f(x)$ is $\mathrm{FP}^{\mathrm{A}[\log n]_{-}}$ hard iff for every function $g(x)$ in $\mathrm{FP}^{\mathrm{A}[\log n]}$ there exist polynomially computable functions $R, S: \Sigma^{*} \rightarrow \Sigma^{*}$ (where $\Sigma$ is the common alphabet) such that $g(x)=S(f(R(x))$ ). A function $f(x)$ is complete in $\mathrm{FP}^{\mathrm{A}[\log n]}$ iff it is in $\mathrm{FP}^{\mathrm{A}[\log n]}$ and is $\mathrm{FP}^{\mathrm{A}[\log n]}$-hard. We show that computing the degree of responsibility of $X=x$ for $\varphi$ in arbitrary models is $\mathrm{FP}^{\Sigma_{2}^{P}[\log n]}$-complete. Similar proof techniques were used by Krentel (1988) to characterize the complexity of various optimization problems. It is shown in (Chockler et al., 2003) that computing the degree of responsibility in binary models is $\mathrm{FP}^{\mathrm{NP}[\log n]}$-complete.

Since there are no known natural $\mathrm{FP}_{2}^{\Sigma_{2}^{P}[\log n]}$-complete problems, the first step in showing that computing the degree of responsibility is $\mathrm{FP}^{\Sigma_{2}^{P}[\log n]}-\operatorname{complete}$ is to define an $\mathrm{FP}_{2}^{\Sigma_{2}^{P}[\log n]_{-}}$

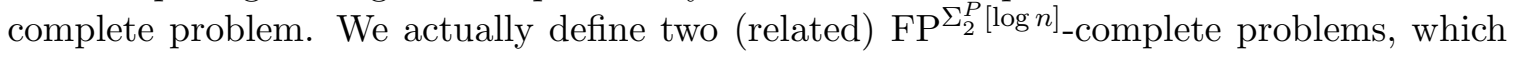
we call MAXQSAT 2 and MINQSAT 2 .

Recall that a quantified Boolean formula (Stockmeyer, 1977) (QBF) has the form $\exists \vec{X}_{1} \forall \vec{X}_{2} \ldots \psi$, where $\vec{X}_{1}, \vec{X}_{2}, \ldots$ are sets of propositional variables and $\psi$ is a propositional formula. A QBF is closed if it has no free propositional variables. TQBF consists of the closed QBF formulas that are true. For example, $\exists \vec{X} \forall \vec{Y}(\vec{X} \Rightarrow \vec{Y}) \in$ TQBF. As shown by Stockmeyer (1977), the following problem $\mathrm{QSAT}_{2}$ is $\Sigma_{2}^{P}$-complete:

$$
\mathrm{QSAT}_{2}=\{\exists \vec{X} \forall \vec{Y} \psi(X, Y) \in \mathrm{TQBF}: \psi \text { is a propositional formula }\} \text {. }
$$

That is, $\mathrm{QSAT}_{2}$ is the language of all true QBFs of the form $\exists \vec{X} \forall \vec{Y} \psi$, where $\psi$ is a quantifierfree propositional formula. 
A witness $f$ for a true closed QBF $\exists \vec{X} \forall \vec{Y} \psi$ is an assignment $f$ to $\vec{X}$ under which $\forall \vec{Y} \psi$ is true. We define $\mathrm{MAXQSAT}_{2}$ as the problem of computing the maximal number of variables in $\vec{X}$ that can be assigned 1 in a witness for $\exists \vec{X} \forall \vec{Y} \psi$. Formally, given a QBF $\Phi=\exists \vec{X} \forall \vec{Y} \psi$, define $\operatorname{MAXQSAT}_{2}(\Phi)$ to be $k$ if there exists a witness for $\Phi$ that assigns exactly $k$ of the variables in $\vec{X}$ the value 1 , and every other witness for $\Phi$ assigns at most $k^{\prime} \leq k$ variables in $\vec{X}$ the value 1 . If $\Phi \notin \mathrm{QSAT}_{2}$, then $\operatorname{MAXQSAT}_{2}(\Phi)=-1$.

Theorem 4.1 MAXQSAT $T_{2}$ is $F P^{\Sigma_{2}^{P}[\log n]}$-complete.

Proof: See the appendix.

Much like MAXQSAT 2 , we define $\operatorname{MINQSAT}_{2}(\exists \vec{X} \forall \vec{Y} \psi)$ to be the minimum number of variables in $\vec{X}$ that can be assigned 1 in a witness for $\exists \vec{X} \forall \vec{Y} \psi$ if there is such a witness, and $|\vec{X}|+1$ otherwise. This problem has the same complexity as $\mathrm{MAXQSAT}_{2}$, as we now show.

Lemma 4.2 MINQSAT $T_{2}$ is $F P^{\Sigma_{2}^{P}[\log n]}$-complete.

Proof: For a propositional formula $\psi$, let $\bar{\psi}$ be the same formula, except that each propositional variable $X$ is replaced by its negation. It is easy to see that

$$
\operatorname{MAXQSAT}_{2}(\exists \vec{X} \forall \vec{Y} \psi)=|\vec{X}|-\operatorname{MINQSAT}_{2}(\exists \vec{X} \forall \vec{Y} \bar{\psi}) .
$$

Thus, MINQSAT 2 and MAXQSAT 2 are polynomially reducible to each other and therefore, by Theorem 4.1, MINQSAT 2 is $\mathrm{FP}_{2}^{\Sigma_{2}^{P}[\log n]}$-complete.

We are now ready to prove $\mathrm{FP}_{2}^{\Sigma_{2}^{P}[\log n]}$-completeness of computing the degree of responsibility for general recursive models.

Theorem 4.3 Computing the degree of responsibility is $F P^{\Sigma_{2}^{P}[\log n]}$-complete in general recursive models.

Proof: We prove the upper bound by describing an algorithm in $\mathrm{FP}_{2}^{\Sigma_{2}^{P}[\log n]}$ for computing the degree of responsibility. For the lower bound, we show that the proof of Eiter and Luakasiewicz (2002a) showing that QSAT $_{2}$ can be reduced to the problem of detecting causality actually provides a reduction from $\mathrm{MINQSAT}_{2}$ to the degree of responsibility.

\subsection{The complexity of computing blame}

Given an epistemic state $(\mathcal{K}, \operatorname{Pr})$, the straightforward way to compute $d b(\mathcal{K}, \operatorname{Pr}, X \leftarrow x, \varphi)$ is by computing is by first computing the degree of responsibility of $X=x$ for $\varphi$ with respect to the situations in $\mathcal{K}$, so as to compute the expected degree of responsibility. Recall that $d r\left(\left(M_{X \leftarrow x}, \vec{u}\right), X=x, \varphi\right)$ can be determined by a binary search that uses at most $\left\lceil\log n_{M}\right\rceil$ queries to the oracle, where $n_{M}$ is the number of endogenous variables in $M$. If there are $N$ situations in $\mathcal{K}$, with $n_{1}, \ldots, n_{N}$ endogenous variables, respectively, this gives a polynomial time algorithm with $\sum_{i=1}^{N}\left\lceil\log n_{i}\right\rceil$ oracle queries. Thus, it is clear that the number of queries is at most the size of the input, and is also at most $N\left\lceil\log n^{*}\right\rceil$, where $n^{*}$ is the maximum 
number of endogenous variables that appear in any of the $N$ situations in $\mathcal{K}$. The type of oracle depends on whether the models are binary or general. For binary models it is enough to have an NP oracle, whereas for general models we need a $\Sigma_{2}^{P}$-oracle.

It follows from the discussion above that the problem of computing the degree of blame in general models $\mathrm{FP}_{2}^{\Sigma_{2}^{P}[n]}$, where $n$ is the size of the input. However, the best lower bound we can prove is $\mathrm{FP}_{2}^{\Sigma_{2}^{P}[\log n]}$, by reducing the problem of computing responsibility to that of computing blame; indeed, the degree of responsibility can be viewed as a special case of the degree of blame with the epistemic state consisting of only one situation. Similarly, lower and upper bounds of $\mathrm{FP}^{\mathrm{NP}[\log n]}$ and $\mathrm{FP}^{\mathrm{NP}[n]}$ hold for binary models.

An alternative characterization of the complexity of computing blame can be given by considering the complexity classes $\mathrm{FP}_{\|}^{\Sigma_{2}^{P}}$ and $\mathrm{FP}_{\|}^{\mathrm{NP}}$, which consist of all functions that can be computed in polynomial time with parallel (i.e., non-adaptive) queries to a $\Sigma_{2}^{P}$ (respectively, NP) oracle. (For background on these complexity classes see (Jenner \& Toran, 1995; Johnson, 1990).) It is easy to see that $\mathrm{FP}_{2}^{\Sigma_{2}^{P}[\log n]} \subseteq \mathrm{FP}_{\|}^{\Sigma_{2}^{P}}$. Indeed, instead of asking $O(\log n)$ queries sequentially (thus adapting each query to the history of answers), the algorithm can ask all possible combinations of queries of the same length in parallel, resulting in a total of $2^{O(\log n)}=O\left(n^{k}\right)$ queries for some constant $k>0$. Using $\mathrm{FP}_{\|}^{\Sigma_{2}^{P}}$ and $\mathrm{FP}_{\|}^{\mathrm{NP}}$, we can get matching upper and lower bounds.

Theorem 4.4 The problem of computing blame in recursive causal models is $F P_{\|_{2}}^{\Sigma_{2}^{P}}$-complete. The problem is $F P_{\|}^{\mathrm{NP}}$-complete in binary causal models.

Proof: See the appendix.

\section{Discussion}

We have introduced definitions of responsibility and blame, based on Halpern and Pearl's definition of causality. We cannot say that a definition is "right" or "wrong", but we can and should examine how useful a definition is, particularly the extent to which it captures our intuitions.

There has been extensive discussion of causality in the philosophy literature, and many examples demonstrating the subtlety of the notion. (These examples have mainly been formulated to show problems with various definitions of causality that have been proposed in the literature.) Thus, one useful strategy for arguing that a definition of causality is useful is to show that it can handle these examples well. This was in fact done for Halpern and Pearl's definition of causality (see (Halpern \& Pearl, 2004a)). While we were not able to find a corresponding body of examples for responsibility and blame in the philosophy literature, there is a large body of examples in the legal literature (see, for example, (Hart \& Honoré, 1985)). We plan to do a more careful analysis of how our framework can be used for legal reasoning in future work. For now, we just briefly discuss some relevant issues.

While we believe that "responsibility" and "blame" as we have defined them are important, distinct notions, the words "responsibility" and "blame" are often used interchangeably in natural language. It is not always obvious which notion is more appropriate in any given situation. For example, Shafer (2001) says that "a child who pokes at a gun's 
trigger out of curiosity will not be held culpable for resulting injury or death". Suppose that a child does in fact poke a gun's trigger and, as a result, his father dies. According to our definition, the child certainly is a cause of his father's death (his father's not leaving the safety catch on might also be a cause, of course), and has degree of responsibility 1. However, under reasonable assumptions about his epistemic state, the child might well have degree of blame 0. So, although we would say that the child is responsible for his father's death, he is not to blame. Shafer talks about the need to take intention into account when assessing culpability. In our definition, we take intention into account to some extent by considering the agent's epistemic state. For example, if the child did not consider it possible that pulling the trigger would result in his father's death, then surely he had no intention of causing his father's death. However, to really capture intention, we need a more detailed modeled of motivation and preferences.

Shafer (2001) also discusses the probability of assessing responsibility in cases such as the following.

Example 5.1 Suppose Joe sprays insecticide on his corn field. It is known that spraying insecticide increases the probability of catching a cold from $20 \%$ to $30 \%$. The cost of a cold in terms of pain and lost productivity is $\$ 300$. Suppose that Joe sprays insecticide and, as a result, Glenn catches a cold. What should Joe pay?

Implicit in the story is a causal model of catching a cold, which is something like the following. ${ }^{2}$ There are four variables:

- a random variable $C$ (for contact) such that $C=1$ if Glenn is in casual contact with someone who has a cold, and 0 otherwise.

- a random variable $I$ (for immune) such that if $I=2$, Glenn does not catch a cold even if he both comes in contact with a cold sufferer and lives near a cornfield sprayed with insecticide; if $I=1$, Glenn does not catch a cold even if comes in contact with a cold sufferer, provided he does not live near a cornfield sprayed with insecticide; and if $I=0$, then Glenn catches a cold if he comes in contact with a cold sufferer, whether or not he lives near a cornfield sprayed with insecticide;

- a random variable $S$ which is 1 if Glenn lives near a cornfield sprayed with insecticide and 0 otherwise;

- a random variable $C C$ which is 1 if Glenn catches a cold and 0 otherwise.

The causal equations are obvious: $C C=1$ iff $C=1$ and either $I=0$ or $I=1$ and $S=1$. The numbers suggest that for $70 \%$ of the population, $I=2$, for $10 \%, I=1$, and for $20 \%$, $I=0$. Suppose that no one (including Glenn) knows whether $I$ is 0,1 , or 2 . Thus, Glenn's expected loss from a cold is $\$ 60$ if Joe does not spray, and $\$ 90$ if he does. The difference of $\$ 30$ is the economic cost to Glenn of Joe spraying (before we know whether Glenn actually has a cold).

As Shafer points out, the law does not allow anyone to sue until there has been damage. So consider the situation after Glenn catches a cold. Once Glenn catches a cold, it is clear

2. This is not the only reasonable causal model that could correspond to the story, but it is good enough for our purposes. 
that $I$ must be either 0 or 1 . Based on the statistical information, $I$ is twice as likely to be 0 as 1 . This leads to an obvious epistemic state, where the causal model where $I=0$ is assigned probability $2 / 3$ and the causal model where $I=1$ is assigned probability $1 / 3$. In the latter model, Joe's spraying is not a cause of Glenn's catching a cold; in the former it is (and has degree of responsibility 1). Thus, Joe's degree of blame for the cold is $1 / 3$. This suggests that, once Joe sprays and Glenn catches a cold, the economic damage is $\$ 100$.

This example also emphasizes the importance of distinguishing between the epistemic states before and after the action is taken, an issue already discussed in Section 3.3. Indeed, examining the situation after Glenn caught a cold enables us to ascribe probability 0 to the situation where Glenn is immune, and thus increases Joe's degree of blame for Glenn's cold.

Example 5.1 is a relatively easy one, since the degree of responsibility is 1 . Things can quickly get more complicated. Indeed, a great deal of legal theory is devoted to issues of responsibility (the classic reference is (Hart \& Honoré, 1985)). There are a number of different legal principles that are applied in determining degree of responsibility; some of these occasionally conflict (at least, they appear to conflict to a layman!). For example, in some cases, legal practice (at least, American legal practice) does not really consider degree of responsibility as we have defined it. Consider the rock throwing example, and suppose the bottle belongs to Ned and is somewhat valuable; in fact, it is worth $\$ 100$.

- Suppose both Suzy and Billy's rock hit the bottle simultaneously, and all it takes is one rock to shatter the bottle. Then they are both responsible for the shattering to degree $1 / 2$ (and both have degree of blame $1 / 2$ if this model is commonly known). Should both have to pay $\$ 50$ ? What if they bear different degrees of responsibility?

Interestingly, a standard legal principle is also that "an individual defendant's responsibility does not decrease just because another wrongdoer was also an actual and proximate cause of the injury" (see (Public Trust, 2003)). That is, our notion of having a degree of responsibility less than one is considered inappropriate in some cases in standard tort law, as is the notion of different degrees of responsibility. Note that if Billy is broke and Suzy can afford $\$ 100$, the doctrine of joint and several liability, also a standard principle in American tort law, rules that Ned can recover the full $\$ 100$ from Suzy.

- Suppose that instead it requires two rocks to shatter the bottle. Should that case be treated any differently? (Recall that, in this case, both Suzy and Billy have degree of responsibility 1.)

- If Suzy's rock hits first and it requires only one rock to shatter the bottle then, as we have seen, Suzy has degree of responsibility 1 or $1 / 2$ (depending on whether we consider only allowable settings) and Billy has degree of responsibility 0 . Nevertheless, standard legal practice would probably judge Billy (in part) responsible.

In some cases, it seems that legal doctrine confounds what we have called cause, blame, and responsibility. To take just one example from Hart and Honoreé (1985, p. 74), assume that $A$ throws a lighted cigarette into the bracken near a forest and a fire starts. Just as the fire is about to go out, $B$ deliberately pours oil on the flame. The fire spreads and burns down the forest. Clearly $B$ 's action was a cause of the forest fire. Was $A$ 's action 
also a cause of the forest fire? According to Hart and Honoré, it is not, whether or not $A$ intended to cause the fire; only $B$ was. In our framework, it depends on the causal model. If $B$ would have started a fire anyway, whether or not $A$ 's fire went out, then $A$ is indeed not the cause; if $B$ would not have started a fire had he not seen $A$ 's fire, then $A$ is a cause (as is $B$ ), although its degree of responsibility for the fire is only $1 / 2$. Furthermore, $A$ 's degree of blame may be quite low. Our framework lets us make distinctions here that seem to be relevant for legal reasoning.

While these examples show that legal reasoning treats responsibility and blame somewhat differently from the way we do, we believe that a formal analysis of legal reasoning using our definitions would be helpful, both in terms of clarifying the applicability of our definitions and in terms of clarifying the basis for various legal judgments. As we said, we hope to focus more on legal issues in future work.

Our notion of degree of responsibility focuses on when an action becomes critical. Perhaps it may have been better termed a notion of "degree of criticality". While we believe that it is a useful notion, there are cases where a more refined notion may be useful. For example, consider a voter who voted for $\mathrm{Mr}$. B in the case of a 1-0 vote and a voter who voted for Mr. B in the case of a 100-99 vote. In both case, that voter has degree of responsibility 1 . While it is true that, in both cases, that voter's vote was critical, in the second case, the voter may believe that his responsibility is more diffuse. We often hear statements like "Don't just blame me; I wasn't the only one who voted for him!" The second author is currently working with I. Gilboa on a definition of responsibility that uses the game-theoretic notion of Shapley value (see, for example, (Osborne \& Rubinstein, 1994)) to try to distinguish these examples.

As another example, suppose that one person dumps 900 pounds of garbage on a porch and another dumps 100 pounds. The porch can only bear 999 pounds of load, so it collapses. Both people here have degree of responsibility $1 / 2$ according to our definition, but there is an intuition that suggests that the person who dumped 900 pounds should bear greater responsibility. We can easily accommodate this in our framework by putting weights on variables. If we use $w t(X)$ to denote the weight of a $X$ and $w t(\vec{W})$ to denote the sum of the weights of variables in the set $\vec{W}$, then we can define the degree of responsibility of $X=x$ for $\varphi$ to be $w t(X) /(w t(\vec{W})+w t(X))$, where $\vec{W}$ is a set of minimal weight for which AC2 holds. This definition agrees with the one we use if the weights of all variables are 1 , so this can be viewed as a generalization of our current definition.

The issue of weights is closely related to another issue, which is the choice of random variables. As pointed out by Halpern and Pearl (2004a), whether $A$ is a cause of $B$ is quite sensitive to the causal model chosen including, among other things, the choice of random variables. This is even more the case for the notions of responsibility and blame. Consider Example 3.3 again, where Mr. B wins an election against Mr. G by a vote of $11-0$. If we represent the situtation as was done in Example 3.3, with 11 random variables, one for each voter, and one additional random variable for the outcome, then he degree of responsibility of $X_{i}=1$ for the outcome is $1 / 6$. However, suppose that instead we represent the voters using two random variables, $X_{1}$ and $Y . X_{1}$, as before, represents voter $1 ; Y$ represents the other 10 voters, so its range has the form $\left(y_{1}, \ldots, y_{10}\right)$, where $y_{j} \in\{0,1\}$ for $j=1, \ldots, 10$. If $Y=(0,1, \ldots, 1)$, for example, then voter two voted for Mr. G, while the remaining 10 voters voted for Mr. B. With this representation, in the case that the vote is $11-0$, the degree 
of responsibility of $X_{1}=1$ for the outcome is $1 / 2$, not $1 / 6$. It is clear that in this case the problem can be dealt with by weighting the variables appropriately. But this example again emphasizes the importance of careful modeling, and that modelers need to be aware of the impact of the model.

More generally, these examples show that there is much more to be done in clarifying our understanding of responsibility and blame. Because these notions are so central in law and morality, we believe that doing so is quite worthwhile.

Acknowledgment A preliminary version of this paper appeared in the Proceedings of 18th International Joint Conference on Artificial Intelligence (IJCAI), 2003. We thank Michael Ben-Or and Orna Kupferman for helpful discussions. We particularly thank Chris Hitchcock, who suggested a simplification of the definition of blame and pointed out a number of typos and other problems in an earlier version of the paper. Finally, we thank the reviewers of the paper for finding yet more typos and pointing out the need to correct some of the proofs. The second author was supported in part by NSF under grant CTC0208535 and by the DoD Multidisciplinary University Research Initiative (MURI) program administered by ONR under grant N00014-01-1-0795. Much of this work was done while the first author was in Hebrew University of Jerusalem, Israel.

\section{Appendix A. Proofs}

In this section, we provide the proofs of all the results in the paper. For convenience, we restate the results here.

Theorem 4.1 MAXQSAT $T_{2}$ is $F P^{\Sigma_{2}^{P}[\log n]}$-complete.

Proof: First we prove that MAXQSAT 2 is in $\mathrm{FP}_{2}^{\Sigma_{2}^{P}[\log n]}$ by describing an algorithm in $\mathrm{FP}^{\Sigma_{2}^{P}[\log n]}$ for solving MAXQSAT 2 . The algorithm queries an oracle $O_{\mathcal{L}}$ for the language $\mathcal{L}$, defined as follows:

$$
\mathcal{L}=\left\{(\Phi, k): k \geq 0, M A X Q S A T_{2}(\Phi) \geq k\right\}
$$

It is easy to see that $\mathcal{L} \in \Sigma_{2}^{P}$ : if $\Phi$ has the form $\exists \vec{X} \forall \vec{Y} \psi$, guess an assignment $f$ that assigns at least $k$ variables in $\vec{X}$ the value 1 and check whether $f$ is a witness for $\Phi$. Note that this amounts to checking the validity of $\psi$ with each variable in $\vec{X}$ replaced by its value according to $f$, so this check is in co-NP, as required. It follows that the language $\mathcal{L}$ is in $\Sigma_{2}^{P}$. (In fact, it is only a slight variant of $\mathrm{QSAT}_{2}$.) Given $\Phi$, the algorithm for computing $\operatorname{MAXQSAT}_{2}(\Phi)$ first checks if $\Phi \in \mathrm{QSAT}_{2}$ by making a query with $k=0$. If it is not, then $\operatorname{MAXQSAT}_{2}(\Phi)$ is -1 . If it is, the algorithm performs a binary search for its value, each time dividing the range of possible values by 2 according to the answer of $O_{\mathcal{L}}$. The number of possible values of $\operatorname{MAXQSAT}_{2}(\Phi)$ is then $|X|+2$ (all values between -1 and $|X|$ are possible), so the algorithm asks $O_{\mathcal{L}}$ at most $\lceil\log n\rceil+1$ queries.

Now we show that MAXQSAT 2 is $\mathrm{FP}^{\Sigma_{2}^{P}[\log n]}$-hard by describing a generic reduction from a problem in $\mathrm{FP}^{\Sigma_{2}^{P}[\log n]}$ to $\mathrm{MAXQSAT}_{2}$. Let $f:\{0,1\}^{*} \rightarrow\{0,1\}^{*}$ be a function in $\mathrm{FP}^{\Sigma_{2}^{P}[\log n]}$. That is, there exist constants $c>0$ and $d>0$ and a deterministic oracle Turing machine $M_{f}$ that on input $w$ outputs $f(w)$ for each $w \in \Sigma^{*}$, and, for all sufficiently large 
inputs $w$, operates in time at most $|w|^{c}$ and queries an oracle for the language $\mathrm{QSAT}_{2}$ at most $d \log |w|$ times. We now describe a reduction from $f$ to $\mathrm{MAXQSAT}_{2}$. Since this is a reduction between function problems, we have to give two polynomial-time functions $r$ and $s$ such that for every input $w$, we have that $r(w)$ is a QBF and $s\left(\operatorname{MAXQSAT}_{2}(r(w))\right)=f(w)$.

We start by describing a deterministic polynomial Turing machine $M_{r}$ that on input $w$ computes $r(w)$. On input $w$ of length $n, M_{r}$ starts by simulating $M_{f}$ on $w$. At some step during the simulation $M_{f}$ asks its first oracle query $q_{1}$. The query $q_{1}$ is a QBF $\exists \vec{Y}_{1} \forall \vec{Z}_{1} \psi_{1}$. The machine $M_{r}$ cannot figure out the answer to $q_{1}$, thus it writes $q_{1}$ down and continues with the simulation. Since $M_{r}$ does not know the answer, it has to simulate the run of $M_{f}$ for both possible answers of the oracle. The machine $M_{r}$ continues in this fashion, keeping track of all possible executions of $M_{f}$ on $w$ for each sequence of answers to the oracle queries. Note that a sequence of answers to oracle queries uniquely characterizes a specific execution of $M_{f}$ (of length $n^{c}$ ). Since there are $2^{d \log n}=n^{d}$ possible sequences of answers, $M_{r}$ on $w$ has to simulate $n^{d}$ possible executions of $M_{f}$ on $w$, thus the running time of this step is bounded by $O\left(n^{c+d}\right)$.

The set of possible executions of $M_{f}$ can be viewed as a tree of height $d \log n$ (ignoring the computation between queries to the oracle). There are at most $n^{d+1}$ queries on this tree. These queries have the form $\exists \vec{Y}_{i} \forall \vec{Z}_{i} \psi_{i}$, for $i=1, \ldots, n^{d+1}$. We can assume without loss of generality that these formulas involve pairwise disjoint sets of variables.

Each execution of $M_{f}$ on $w$ involves at most $d \log n$ queries from this collection. Let $X_{j}$ be a variable that describes the answer to the $j$ th query in an execution, for $1 \leq j \leq d \log n$. (Of course, which query $X_{j}$ is the answer to depends on the execution.) Each of the $n^{d}$ possible assignments to the variables $X_{1}, \ldots, X_{d \log n}$ can be thought of as representing a number $a \in\left\{0, \ldots, n^{d}-1\right\}$ in binary. Let $\zeta_{a}$ be the formula that characterizes the assignment to these variables corresponding to the number $a$. That is, $\zeta_{a}=\wedge_{j=1}^{d \log n} X_{j}^{a}$, where $X_{j}^{a}$ is $X_{j}$ if the $j$ th bit of $a$ is 1 in $a$ and $\neg X_{j}$ otherwise. Under the interpretation of $X_{j}$ as determining the answer to the query $j$, each such assignment $a$ determines an execution of $M_{f}$. Note that the assignment corresponding to the highest number $a$ for which all the queries corresponding to bits of $a$ that are 1 are true is the one that corresponds to the actual execution of $M_{f}$. For suppose that this is not the case. That is, suppose that the actual execution of $M_{f}$ corresponds to some $a^{\prime}<a$. Since $a^{\prime}<a$, there exist bits on which $a$ and $a^{\prime}$ disagree. Let $X_{j}$ be the most significant bit on which $a$ and $a^{\prime}$ disagree. Since $a^{\prime}<a$, we have that $X_{j}$ is 0 in $a^{\prime}$ and is 1 in $a$. By our choice of $a$, the query $\exists \vec{Y}_{i} \forall \vec{Z}_{i} \psi$ corresponding to $X_{j}$ is a true $\mathrm{QBF}$, thus a $\Sigma_{2}^{P}$-oracle that $M_{f}$ queries should have answered "yes" to this query, contradicting the assumption that $X_{j}=0$.

The next formula provides the connection between the $X_{i}$ s and the queries. It says that if $\zeta_{a}$ is true, then all the queries corresponding to the bits that are 1 in $a$ must be true QBF. For each assignment $a$, let $a_{1}, \ldots, a_{N_{a}}$ be the queries that were answered "YES" during the execution of the $M_{f}$ corresponding to $a$. Note that $N_{a} \leq d \log n$. Define

$$
\eta=\bigwedge_{a=0}^{n^{d}-1}\left(\zeta_{a} \Rightarrow \exists \vec{Y}_{a_{1}} \forall \vec{Z}_{a_{1}} \psi_{a_{1}} \wedge \ldots \exists \vec{Y}_{a_{N_{a}}} \forall \vec{Z}_{a_{\log n}} \psi_{a_{N_{a}}}\right)
$$

The variables $\vec{Y}_{a_{i}}, \vec{Z}_{a_{i}}$ do not appear in the formula $\eta$ outside of $\psi_{a_{i}}$, thus we can re-write $\eta$ as

$$
\eta=\exists \vec{Y}_{1}, \ldots, \vec{Y}_{n^{d}}, \ldots, \forall \vec{Z}_{1}, \ldots, \vec{Z}_{n^{d}} \eta^{\prime}
$$


where

$$
\eta^{\prime}=\bigwedge_{a=0}^{n^{d}-1} \zeta_{a} \Rightarrow\left(\psi_{a_{1}} \wedge \ldots \wedge \psi_{a_{d \log n}}\right)
$$

The idea now is to use MAXQSAT 2 to compute the highest-numbered assignment $a$ such that all the queries corresponding to bits that are 1 in $a$ are true. To do this, it is useful to express the number $a$ in unary. Let $U_{1}, \ldots, U_{n^{d}}$ be fresh variables. We introduce the following convention for representing numbers in unary notation using these variables: $U_{1}, \ldots, U_{n^{d}}$ represent a number $j$ in unary if $U_{i}=1$ for each $i \leq j$ and $U_{i}=0$ for each $i>j$. Let $\xi$ express the fact that the $U_{i}$ s represent the same number as $X_{1}, \ldots, X_{d \log n}$, but in unary:

$$
\xi=\bigwedge_{i=1}^{n^{d}}\left(\neg U_{i} \Rightarrow \neg U_{i+1}\right) \bigwedge\left(\neg U_{1} \Rightarrow\left(X_{1}^{0} \wedge \ldots \wedge X_{d \log n}^{0}\right)\right) \wedge \bigwedge_{a=1}^{n^{d}-1}\left[\left(U_{a} \wedge \neg U_{a+1}\right) \Rightarrow\left(X_{1}^{a} \wedge \ldots \wedge X_{d \log n}^{a}\right)\right] .
$$

Let $\Phi=\exists U_{1}, \ldots, U_{n^{d}}, X_{1}, \ldots, X_{d \log n}(\eta \wedge \xi)$. Note that the size of $\Phi$ is polynomial in $n$ and, for each $i$, the variables $\vec{Y}_{i}$ and $\vec{Z}_{i}$ do not appear in $\varphi$ outside of $\eta$. Thus, we can rewrite $\Phi$ in the following form:

$$
\Phi=\exists U_{1}, \ldots, U_{n^{d}}, X_{1}, \ldots, X_{d \log n}, \vec{Y}_{1}, \ldots, \vec{Y}_{n^{d}} \forall \vec{Z}_{1}, \ldots, \vec{Z}_{n^{d}}\left(\eta^{\prime} \wedge \xi\right) .
$$

Thus, $\Phi$ has the form $\exists \vec{X} \forall \vec{Y} \psi$.

We are interested in the witness for $\Phi$ that makes the most $U_{i}$ 's true, since this will tell us the execution actually followed by $M_{f}$. While MAXQSAT 2 gives us the maximal number of variables that can be assigned true in a witness for $\Phi$, this is not quite the information we need. Assume for now that we have a machine that is capable of computing a slightly generalized version of MAXQSAT 2 . We denote this version by SUBSET_MAXQSAT 2 and define it as a maximal number of 1's in a witness computed for a given subset of variables (and not for the whole set as MAXQSAT 2 ). Formally, given a QBF $\Psi=\exists \vec{X} \forall \vec{Y} \psi$ and a set $\vec{Z} \subseteq \vec{X}$, we define SUBSET_MAXQSAT $2(\Psi, \vec{Z})$ as the maximal number of variables from $\vec{Z}$ assigned 1 in a witness for $\Psi$, or -1 if $\Psi$ is not in TQBF.

Clearly, SUBSET_MAXQSAT $2(\Phi, \vec{U})$ gives us the assignment which determines the execution of $M_{f}$. Let $r(w)=\Phi$. Let $s$ be the function that extracts $f(w)$ from SUB$\operatorname{SET}_{-} \operatorname{MAXQSAT}_{2}\left(\Phi,\left\{U_{1}, \ldots, U_{n}\right\}\right)$. (This can be done in polynomial time, since $f(w)$ can be computed in polynomial time, given the answers to the oracle.)

It remains to show how to reduce SUBSET_MAXQSAT 2 to $\mathrm{MAXQSAT}_{2}$. Given a formula $\Psi=\exists \vec{X} \forall \vec{Y} \psi$ and a set $\vec{Z} \subseteq \vec{X}$, we compute $\operatorname{SUBSET}_{-} \operatorname{MAXQSAT}_{2}(\Psi, \vec{Z})$ in the following way. Let $\vec{U}=\vec{X} \backslash \vec{Z}$. For each $U_{i} \in \vec{U}$ we add a variable $U_{i}^{\prime}$. Let $\vec{U}^{\prime}$ be the set of all the $U_{i}^{\prime}$ variables. Define the formula $\Psi^{\prime}$ as $\exists \vec{X} \overrightarrow{U^{\prime}} \forall \vec{Y}\left(\psi \wedge \wedge_{U_{i} \in \vec{U}}\left(U_{i} \Leftrightarrow \neg U_{i}^{\prime}\right)\right)$. Clearly, in all consistent assignments to $\vec{X} \cup \vec{U}^{\prime}$ exactly half of the variables in $\vec{U} \cup \vec{U}^{\prime}$ are assigned 1. Thus, the witness that assigns $\operatorname{MAXQSAT}_{2}\left(\Psi^{\prime}\right)$ variables the value 1 also assigns $\operatorname{SUBSET}_{-} \mathrm{MAXQSAT}_{2}(\Psi, \vec{Z})$ variables from $\vec{Z}$ the value 1 . The value SUB$\operatorname{SET}_{\operatorname{MAXQSAT}}(\Psi, \vec{Z})$ is computed by subtracting $|\vec{U}|$ from $\operatorname{MAXQSAT}_{2}\left(\Psi^{\prime}\right)$.

Theorem 4.3 Computing the degree of responsibility is $F P^{\Sigma_{2}^{P}[\log n]}$-complete in general recursive models. 
Proof: First we prove membership in $\mathrm{FP}^{\Sigma_{2}^{P}[\log n]}$ by describing an algorithm in $\mathrm{FP}_{2}^{\Sigma_{2}^{P}[\log n]}$ for computing the degree of responsibility. Consider the oracle $O_{\mathcal{L}}$ for the language $\mathcal{L}$, defined as follows:

$$
\mathcal{L}=\{\langle(M, \vec{u}),(X=x), \varphi, i\rangle: 0 \geq i \geq 1, \text { and } d r((M, \vec{u}),(X=x), \varphi) \geq i\} .
$$

In other words, $\langle(M, \vec{u}),(X=x), \varphi, i\rangle \in \mathcal{L}$ for $i>0$ if there is a partition $(\vec{Z}, \vec{W})$ and setting $\left(x^{\prime}, \vec{w}^{\prime}\right)$ satisfying condition AC2 in Definition 3.1 such that at most $(1 / i)-1$ variables in $W$ have values different in $\vec{w}^{\prime}$ from their value in the context $\vec{u}$. It is easy to see that $\mathcal{L} \in \Sigma_{2}^{P}$. Given as input a situation $(M, \vec{u}), X=x$ (where $X$ is an endogenous variable in $M$ and $x \in \mathcal{R}(X)$ ), and a formula $\varphi$, the algorithm for computing the degree of responsibility uses the oracle to perform a binary search on the value of $\operatorname{dr}((M, \vec{u}),(X=x), \varphi)$, each time dividing the range of possible values for the degree of responsibility by 2 according to the answer of $O_{\mathcal{L}}$. The number of possible candidates for the degree of responsibility is $n-|X|+1$, where $n$ is the number of endogenous variables in $M$. Thus, the algorithm runs in linear time (and logarithmic space) in the size of the input and uses at most $\lceil\log n\rceil$ oracle queries. Note that the number of oracle queries depends only on the number of endogenous variables in the model, and not on any other features of the input.

As we said in the main part of the text, the proof that computing the degree of responsibility is $\mathrm{FP}_{2}^{\Sigma_{2}^{P}[\log n]}$-hard essentially uses the same argument used by Eiter and Luakasiewicz (2002a) to show that $\mathrm{QSAT}_{2}$ can be reduced to the problem of detecting causality, together with an argument that their proof actually provides a reduction from MINQSAT $_{2}$ to the degree of responsibility. We now describe the reduction in more detail. Given as input a closed QBF $\Phi=\exists \vec{A} \forall \vec{B} \varphi$, where $\varphi$ is a propositional formula, define the situation $(M, \vec{u})$ as follows. $M=((\mathcal{U}, \mathcal{V}, \mathcal{R}), \mathcal{F})$, where

- $\mathcal{U}$ consists of the single variable $E$;

- $\mathcal{V}$ consists of the variables $\vec{A} \cup \vec{B} \cup\{C, X\}$, where $C$ and $X$ are fresh variables;

- $\mathcal{R}$ is defined so that each variable $S \in \vec{A} \cup\{C, X\}$ has range $\mathcal{R}(S)=\{0,1\}$, while each variable $S \in \vec{B}$ has range $\mathcal{R}(S)=\{0,1,2\}$;

- $\mathcal{F}$ is defined so that $F_{S}=C+X$ for $S \in \vec{B}$ and $F_{S}=0$ for $S \in \mathcal{V}-\vec{B}$.

Let $\vec{u}$ be the context in which $E$ is set to 0 . Note that every variable in $\mathcal{V}$ has value 0 in context $\vec{u}$. (This would also be true in the context where $E$ is set to 1 ; the context plays no role here.) Let

$$
\psi=\left(\varphi^{\prime} \wedge \bigwedge_{S \in \vec{B}} S \neq 2\right) \vee(C=0) \vee\left(X=1 \wedge C=1 \wedge \bigvee_{S \in \vec{B}} S \neq 2\right)
$$

where $\varphi^{\prime}$ is obtained from $\varphi$ by replacing each variable $S \in \vec{A} \cup \vec{B}$ in $\varphi$ by $S=1$. It is shown in (Eiter \& Lukasiewicz, 2002a) that $X=0$ is a cause of $\psi$ in $(M, \vec{u})$ iff $\Phi \in$ TQBF. We now extend their argument to show that the degree of responsibility of $X=0$ for $\psi$ is actually $1 /\left(\operatorname{MINQSAT}_{2}(\Phi)+2\right)$.

Suppose first that $X=0$ is a cause of $\psi$ in $(M, \vec{u})$. Then there exists a partition $(\vec{Z}, \vec{W})$ of $\mathcal{V}$ and a setting $\vec{w}$ of $\vec{W}$ such that $(M, u) \models[X \leftarrow 1, \vec{W} \leftarrow \vec{w}] \neg \psi$ and $(M, u) \models[X \leftarrow$ 
$\left.0, \vec{W} \leftarrow \vec{w}, \vec{Z}^{\prime} \leftarrow \overrightarrow{0}\right] \psi$, where $\vec{Z}^{\prime}$ is as in $\mathrm{AC} 2(\mathrm{~b})$. (Since every variable gets value 0 in context $\vec{u}$, the $\vec{z}^{*}$ of $\mathrm{AC} 2(\mathrm{~b})$ becomes $\overrightarrow{0}$ here.) Suppose that $\vec{W}$ is a minimal set that satisfies these conditions.

Eiter and Lukasiewicz show that $\vec{B} \cap \vec{W}=\emptyset$. To prove this, suppose that $S \in \vec{B} \cap \vec{W}$. If $S$ is set to either 0 or 1 in $\vec{w}$, then $(M, u) \forall \forall[\vec{W} \leftarrow \vec{w}, X \leftarrow 1] \neg \psi$, since the clause $\left(X=1 \wedge C=1 \wedge \bigvee_{S \in B} S \neq 2\right.$ ) will be satisfied. On the other hand, if $S$ is set to 2 in $w$, then $(M, u) \forall \forall[\vec{W} \leftarrow \vec{w}, X \leftarrow 0] \psi$. Thus, $\vec{B} \cap \vec{W}=\emptyset$. Moreover, $C \in \vec{W}$, since $C$ has to be set to 1 in order to falsify $\psi$. It follows that $\vec{W} \subseteq \vec{A} \cup\{C\}$.

Note that every variable in $\vec{W}$ is set to $1 \mathrm{in} \vec{w}$. This is obvious for $C$. If there is some variable $S$ in $\vec{W} \cap \vec{A}$ that is set to 0 in $\vec{w}$, we could take $S$ out of $\vec{W}$ while still satisfying condition AC2 in Definition 3.1, since every variable $S$ in $\vec{W} \cap \vec{A}$ has value 0 in context $\vec{u}$ and can only be 1 if it is set to 1 in $\vec{w}$. This contradicts the minimality of $W$. To show that $\Phi \in \mathrm{TQBF}$, consider the truth assignment $v$ that sets a variable $S \in \vec{A}$ true iff $S$ is set to 1 in $\vec{W}$. It suffices to show that $v \models \forall \vec{B} \varphi$. Let $v^{\prime}$ be any truth assignment that agrees with $v$ on the variables in $\vec{A}$. We must show that $v^{\prime}=\varphi$. Let $\vec{Z}^{\prime}$ consist of all variables $S \in \vec{B}$ such that $v^{\prime}(S)$ is false. Since $(M, u) \models\left[X \leftarrow 0, \vec{W} \leftarrow \overrightarrow{1}, \vec{Z}^{\prime} \leftarrow \overrightarrow{0}\right] \psi$, it follows that $(M, u) \models\left[X \leftarrow 0, \vec{W} \leftarrow \overrightarrow{1}, \vec{Z}^{\prime} \leftarrow \overrightarrow{0}\right] \varphi^{\prime}$. It is easy to check that if every variable in $\vec{W}$ is set to $1, X$ is set to 0 , and $\vec{Z}^{\prime}$ is set to $\overrightarrow{0}$, then every variable in $\vec{A} \cup \vec{B}$ gets the same value as it does in $v^{\prime}$. Thus, $v^{\prime} \models \varphi$. It follows that $\Phi \in \mathrm{TQBF}$. This argument also shows that there is a witness for $\Phi$ (i.e., valuation which makes $\forall \vec{B} \varphi$ true) that assigns the value true to all the variables in $\vec{W} \cap \vec{A}$, and only to these variables.

Now suppose that if $\Phi \in \mathrm{TQBF}$ and let $v$ be a witness for $\Phi$. Let $\vec{W}$ consist of $C$ together with the variables $S \in \vec{A}$ such that $v(S)$ is true. Let $\vec{w}$ set all the variables in $\vec{W}$ to 1 . It is easy to see $X=0$ is a cause of $\psi$, using this choice of $\vec{W}$ and $\vec{w}$ for AC2. Clearly $(M, u) \models[X \leftarrow 1, \vec{W} \leftarrow \overrightarrow{1}] \neg \psi$, since setting $X$ to 1 and and all variables in $\vec{W}$ to 1 causes all variables in $\vec{B}$ to have the value 2 . On the other hand, $(M, u) \models\left[X \leftarrow 0, \vec{W} \leftarrow \overrightarrow{1}, \vec{Z}^{\prime} \leftarrow 0\right] \psi$, since setting $X$ to 0 and $\vec{W} \leftarrow \vec{w}$ guarantees that all variables in $\vec{B}$ have the value 1 and $\varphi^{\prime}$ is satisfied. It now follows that a minimal set $\vec{W}$ satisfying AC2 consists of $C$ together with a minimal set of variables in $\vec{A}$ that are true in a witness for $\Phi$. Thus, $|\vec{W}|=\operatorname{MINQSAT}_{2}(\Phi)+$ 1 , and we have $d r((M, \vec{u}),(X=0), \psi)=1 /(|\vec{W}|+1)=1 /\left(\operatorname{MINQSAT}_{2}(\Phi)+2\right)$.

Theorem 4.4 The problem of computing blame in recursive causal models is $F P_{\|}^{\Sigma_{2}^{P}}$-complete. The problem is $F P_{\|}^{\mathrm{NP}}$-complete in binary causal models.

Proof: As we have observed, the naive algorithm for computing the degree of blame uses $N \log n^{*}$ queries, where $N$ is the number of situations in the epistemic state, and $n^{*}$ is the maximum number of variables in each one. However, the answers to oracle queries for one situation do not affect the choice of queries for other situations, thus queries for different situations can be asked in parallel. The queries for one situation are adaptive, however, as shown by Jenner and Toran (1995), a logarithmic number of adaptive queries can be replaced with a polynomial number of non-adaptive queries by asking all possible combinations of queries in parallel. Thus, the problem of computing blame is in $\mathrm{FP}_{\|}^{\Sigma_{2}^{P}}$ for arbitrary recursive models, and in $\mathrm{FP}_{\|}^{\mathrm{NP}}$ for binary causal models. 
For hardness in the case of arbitrary recursive models, we provide a reduction from the following $\mathrm{FP}_{\|}^{\Sigma_{2}^{P}}$-complete problem (Jenner \& Toran, 1995; Eiter \& Lukasiewicz, 2002a). Given $N$ closed QBFs $\Phi_{1}, \ldots, \Phi_{N}$, where $\Phi_{i}=\exists \vec{A}_{i} \forall \vec{B}_{i} \varphi_{i}$ and $\varphi_{i}$ is a propositional formula of size $O(n)$ for $1 \leq i \leq N$, compute the vector $\left(v_{1}, \ldots, v_{N}\right) \in\{0,1\}^{N}$ such that for all $i \in\{1, \ldots, N\}$, we have that $v_{i}=1$ iff $\Phi_{i} \in T Q B F$. Without loss of generality, we assume

that all $\vec{A}_{i}$ 's and $\vec{B}_{i}$ 's are disjoint. We construct an epistemic state $(\mathcal{K}, \operatorname{Pr})$, a formula $\varphi$, and a variable $X$ such that $\left(v_{1}, \ldots, v_{N}\right)$ can be computed in polynomial time from the degree of blame of setting $X$ to 1 for $\varphi$ relative to $(\mathcal{K}, \operatorname{Pr})$. $\mathcal{K}$ consists of the $N+1$ situations $\left(M_{i}, \vec{u}_{i}\right), i=1, \ldots, N+1$. Each of the models $M_{1}, \ldots, M_{N+1}$ is of size $O(N \cdot n)$ and involves all the variables that appear in the causal models constructed in the hardness part of the proof of Theorem 4.3, together with fresh random variables num $_{1}, \ldots, n u m_{N+1}$. For $1 \leq i \leq N$, the equations for the variables in $\Phi_{i}$ in the situation $\left(M_{i}, \vec{u}_{i}\right)$ are the same as in the proof of Theorem 4.3. Thus, $\Phi_{i} \in \mathrm{TQBF}$ iff $X=1$ is a cause of $\varphi_{i}$ in $\left(M_{i}, \vec{u}_{i}\right)$; moreover, if $X=1$ is a cause of $\varphi_{i}$, then it has degree of responsibility 1. In addition, for $i=1, \ldots, n$, in $\left(M_{i}, \vec{u}_{i}\right)$, the equations are such that num $_{i}$ and $n u m_{N+1}$ are set to 1 and all other variables (i.e., num $_{j}$ for $j \notin\{i, N+1\}$ and all the variables in $\Phi_{j}, j \neq i$ ) are set to 0 . The situation $\left(M_{N+1}, \vec{u}_{N+1}\right)$ is such that all variables are set to 0 at both times 0 and 1 . Let $\operatorname{Pr}\left(M_{i}, \vec{u}_{i}\right)=1 / 2^{i(\lceil\log n\rceil)}$ for $1 \leq i \leq N$, and let $\operatorname{Pr}\left(M_{N+1}, \vec{u}_{N+1}\right)=1-\Sigma_{i=1}^{N} 1 / 2^{i(\lceil\log n\rceil)}$. Finally, let $\varphi=\bigwedge_{i=1}^{N}\left(\right.$ num $\left._{i} \rightarrow \varphi_{i}\right) \wedge$ num $_{N+1}$.

Clearly $\varphi$ is true in $\left(M_{i}, \vec{u}_{i}\right), i=1, \ldots, N$, iff $X=1$ is a cause of $\varphi_{i}$; moreover, if $X=1$ is a cause of $\varphi_{i}$, then $X=1$ has degree of responsibility 1 . In addition, $\varphi$ is false in $\left(M_{N+1}, \vec{u}_{N+1}\right)$. Thus, the degree of blame $d b(\mathcal{K}, \operatorname{Pr}, X \leftarrow x, \varphi)$ is an $N\lceil\log n\rceil$-bit fraction, where the $i$ th group of bits of size $n$ is not 0 iff $\Phi_{i} \in$ TQBF. It immediately follows that the vector $\left(v_{1}, \ldots, v_{N}\right)$ can be extracted from $d b(\mathcal{K}, \operatorname{Pr}, X \leftarrow x, \varphi)$ by assigning $v_{i}$ the value 1 iff the bits in the $i$ th group in $d b(\mathcal{K}, \operatorname{Pr}, X \leftarrow x, \varphi)$ are not all 0 .

We can similarly prove that computing degree of blame in binary models is $\mathrm{FP}_{\|}^{\mathrm{NP}}$-hard by reduction from the problem of determining which of $N$ given propositional formulas of size $O(n)$ are satisfiable. We leave the details to the reader.

\section{References}

Chockler, H., Halpern, J. Y., \& Kupferman, O. (2003). What causes a system to satisfy a specification?. Unpublished manuscript. Available at http://www.cs.cornell.edu/home/halpern/papers/resp.ps.

Collins, J., Hall, N., \& Paul, L. A. (Eds.). (2004). Causation and Counterfactuals. MIT Press, Cambridge, Mass.

Eiter, T., \& Lukasiewicz, T. (2002a). Causes and explanations in the structural-model approach: tractable cases. In Proc. Eighteenth Conference on Uncertainty in Artificial Intelligence (UAI 2002), pp. 146-153.

Eiter, T., \& Lukasiewicz, T. (2002b). Complexity results for structure-based causality. Artificial Intelligence, 142(1), 53-89. 
Hall, N. (2004). Two concepts of causation. In Collins, J., Hall, N., \& Paul, L. A. (Eds.), Causation and Counterfactuals. MIT Press, Cambridge, Mass.

Halpern, J. Y., \& Pearl, J. (2004a). Causes and explanations: A structural-model approach. Part I: Causes. British Journal for Philosophy of Science.

Halpern, J. Y., \& Pearl, J. (2004b). Causes and explanations: A structural-model approach. Part II: Explanations. British Journal for Philosophy of Science.

Hart, H. L. A., \& Honoré, T. (1985). Causation in the Law (Second Edition edition). Oxford University Press, Oxford, U.K.

Hume, D. (1739). A Treatise of Human Nature. John Noon, London.

Jenner, B., \& Toran, J. (1995). Computing functions with parallel queries to NP. Theoretical Computer Science, 141, 175-193.

Johnson, D. (1990). A catalog of complexity classes. In van Leeuwen, J. (Ed.), Handbook of Theoretical Computer Science, Vol. A, chap. 2. Elsevier Science.

Krentel, M. (1988). The complexity of optimization problems. Journal of the CSS, 36, 490-509.

Osborne, M. J., \& Rubinstein, A. (1994). A Course in Game Theory. MIT Press, Cambridge, Mass.

Papadimitriou, C. H. (1984). The complexity of unique solutions. Journal of ACM, 31, $492-500$.

Pearl, J. (2000). Causality: Models, Reasoning, and Inference. Cambridge University Press, New York.

Public Trust (2003). Tort law issues/fact sheets. http://www.citizen.org/congress/civjus/tort/tortlaw/articles.cfm?ID=834).

Shafer, G. (1996). The Art of Causal Conjecture. MIT Press, Cambridge, Mass.

Shafer, G. (2001). Causality and responsibility. Cardozo Law Review, 22, 101-123.

Stockmeyer, L. J. (1977). The polynomial-time hierarchy. Theoretical Computer Science, $3,1-22$.

Zimmerman, M. (1988). An Essay on Moral Responsibility. Rowman and Littlefield, Totowa, N.J. 